\title{
SATELLITE OBSERVATIONS OF NEW PARTICLE AND FIELD SIGNATURES ASSOCIATED WITH SAR ARC FIELD LINES AT MAGNETOSPHERIC HEIGHTS
}

\author{
J. U. Kozyra, ${ }^{1}$ T. E. Cravens, ${ }^{1}$ A. F. Nagy, ${ }^{1}$ D. A. Gurnett,${ }^{2}$ \\ R. L. Huff, ${ }^{2}$ R. H. Comfort ${ }^{3}$ J. H. Waite, Jr, ${ }^{4}$ L. H. Brace, ${ }^{5}$ \\ J. D. Winningham, ${ }^{6}$ J. L. Burch ${ }^{6}$ and W. K. Peterson ${ }^{7}$ \\ ${ }^{I}$ Space Physics Research Laboratory, University of Michigan, Ann Arbor, \\ MI 48109, U.S.A. \\ ${ }^{2}$ University of Iowa, Iowa City, IA 52242, U.S.A. \\ ${ }^{3}$ University of Alabama in Huntsville, Huntsville, AL 35899, U.S.A. \\ ${ }^{4}$ NASA/Marshall Space Flight Center, Huntsville, AL 35812, U.S.A. \\ ${ }^{5}$ NASA/Goddard Space Flight Center, Greenbelt, MD 20771, U.S.A. \\ ${ }^{6}$ Southwest Research Institute, P.O. Drawer 28510, San Antonio, \\ TX 78284, U.S.A. \\ ${ }^{7}$ Lockheed Corporation, Palo Alto, CA 94304, U.S.A.
}

\section{ABSTRACT}

Enhancements in thermal ion densities, an oxygen dominated ring current at energies below $17 \mathrm{kev}$, and invariant latitude-limited bands of intense ELF hiss have been discovered on Stable Auroral Red (SAR) arc field lines at magnetospheric heights. These new signatures were revealed by an examination of 31 coordinated data sets taken simultaneously at magnetospheric and ionospheric heights by the DE-1 and -2 satellites during SAR arc traversals within the period September 1981 through April 1982. Data sets from DE-2, for the first time, provide information on the location of a SAR arc (determined by the F region electron temperature enhancement) during the nearly simultaneous passage of these field lines by DE-1 in the magnetosphere. These new high altitude signatures are examined in the context of possible magnetospheric SAR arc energy source mechanisms.

\section{INTRODUCTION}

Stable Auroral Red (SAR) arcs are ionospheric phenomena, although the source of their energy lies in the magnetosphere. The extended nature of the processes responsible for this enhanced, nearly monochromatic $6300 \AA$ emission has made observational investigations aimed at examining the SAR arc's energy source difficult to pursue. A number of theories have been advanced to explain the source of the SAR arc's energy /1,2,3/ but, to date, none have received sufficient observational support. Ionospheric signatures associated with SAR arcs and proposed energy source mechanisms have been summarized in Rees and Roble /4/ and updated in Kozyra /5/.

Simultaneous or nearly simultaneous observations in the energy sink region at $400-800 \mathrm{~km}$ altitude and in the magnetospheric energy source region tens of thousands of kilometers above this altitude are necessary to evaluate realistically the SAR arc energy source mechanisms. Such data sets were virtually non-existent until the launch of the Dynamics Explorer (DE) satellite pair. The DE satellites were placed into elliptical coplanar polar orbits in August 1981. This unique orbital configuration allows data acquisition at two altitudes along a common magnetic flux tube, making them ideally suited for pursuing an observational investigation of SAR arcs.

\section{DATA SELECTION PROCEDURE}

Data were collected during the eight month period from September 1981 through April 1982 on days during which SAR arcs were observed by the MASP chain of ground-based photometers $/ 6 \%$. A magnetic coincidence was defined, for the purposes of this study, as a time lapse of less than 30 minutes between DE- 1 and DE- 2 traversals of a SAR arc region; this period is small compared to a "typical" SAR arc lifetime. The location of the SAR arc in each data set is defined by the elevated ionospheric electron temperature directly responsible for the $6300 \AA$ emission which characterizes the SAR arc (cf. $/ 4 /$ ).

Thirty-one data sets on 18 different days during the period September 1981 through April 1982 were compiled. These data sets were taken largely between 21 and 23 hours local solar time. Observations at ionospheric heights $/ 5 /$ serve to confirm and clarify signatures that have previously been reported in the literature (c.f. /4/). In addition, these data sets reveal new and interesting signatures in particle and field observations on SAR arc field lines at magnetospheric heights. Results, reported here, will briefly outline the new signatures that are seen at high altitude over SAR arcs. A more in-depth discussion of these signatures and their possible significance is presented elsewhere $\Pi, 8 /$ 

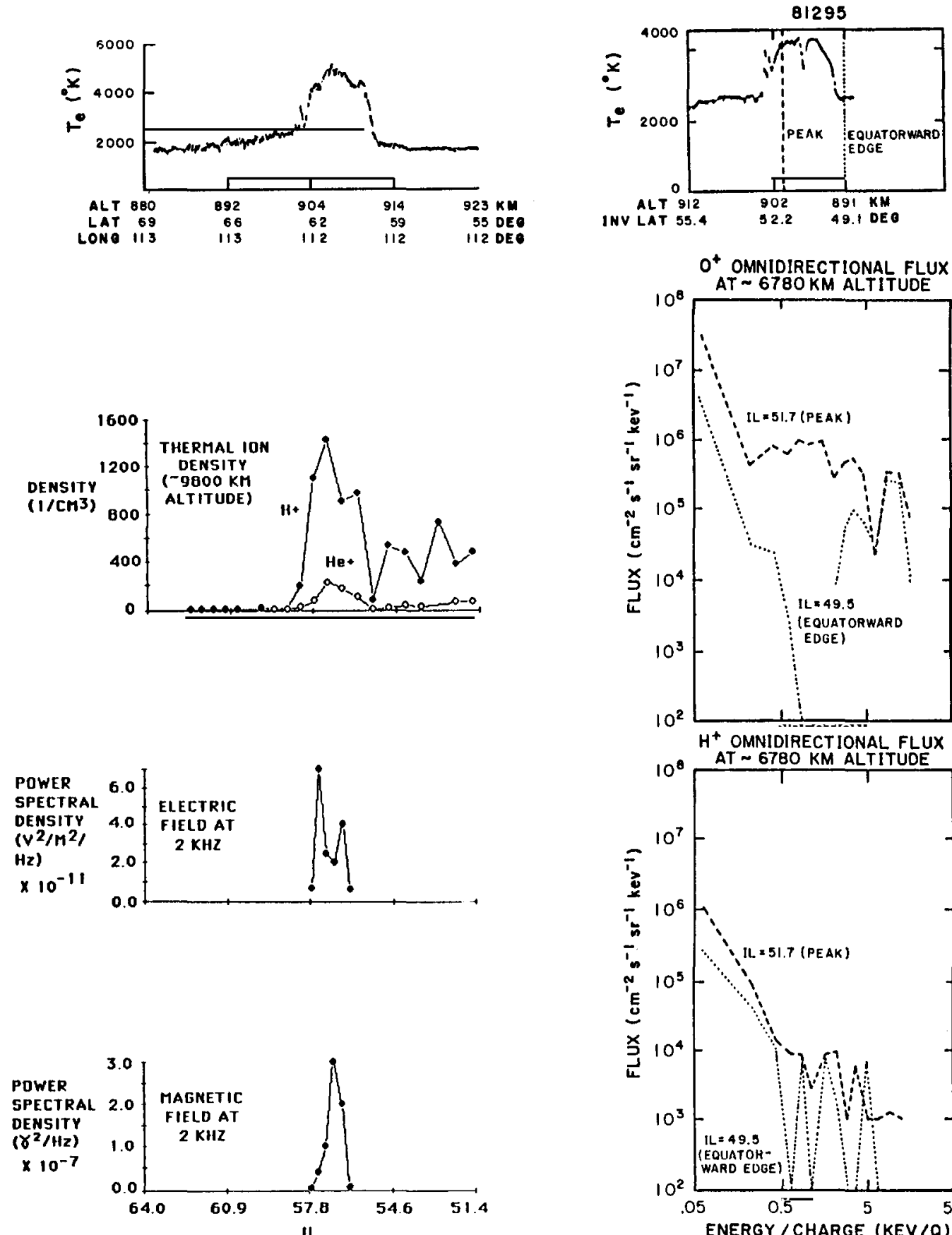

Fig. 1. Observations of the $\mathrm{F}$ region electron temperature enhancement associated with the 8 October 1981 SAR arc are presented in the upper panel. The remaining panels display nearly simultaneous observations of the thermal ion densities and the electric and magnetic fields at $2 \mathrm{KHz}$, respectively, in the magnetosphere on field lines associated with the SAR arc.

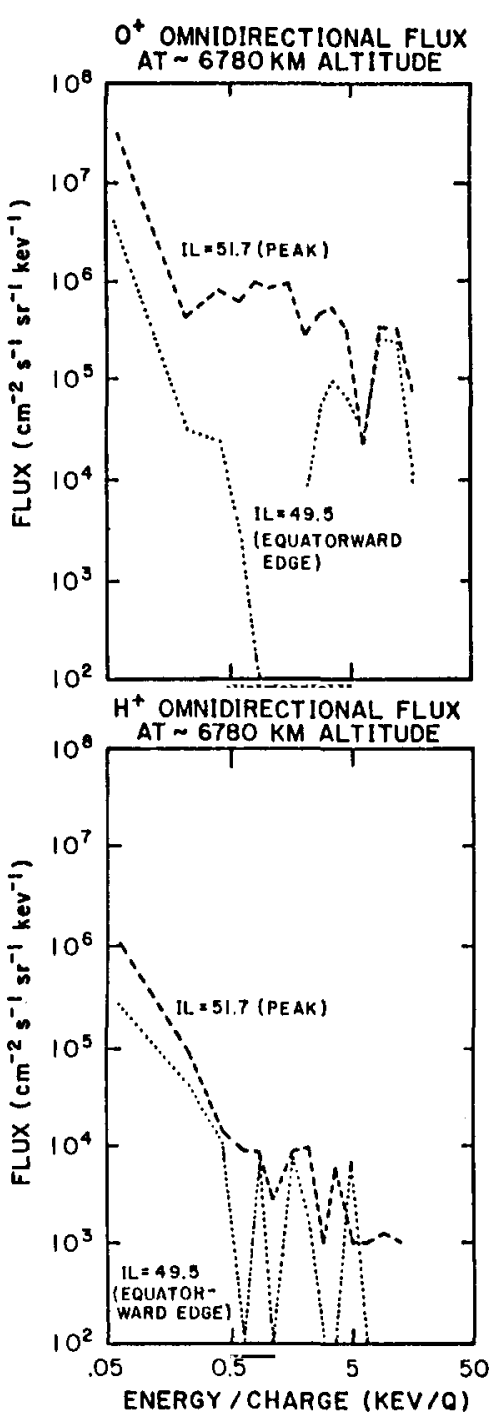

Fig. 2. Observations of the $F$ region electron temperature enhancement associated with the 22 October 1981 SAR arc. The remaining panels display the $\mathrm{O}^{+}$(middle panel) and $\mathrm{H}^{+}$(upper panel) fluxes, averaged over pitch angle, observed at magnetospheric heights on selected field lines associated with the SAR arc. 


\section{OBSERVATIONS}

Figure 1 presents observations taken during the 8 October 1981 SAR arc. The lower satellite (DE-2) crossed SAR arc field lines at approximately $900 \mathrm{~km}$ altitude between 14:04:20 and 14:05:06 UT. Elevated ionospheric electron temperatures measured by the Langmuir probe on DE-2 (upper panel) were used to define the SAR arc field lines, $57.7^{\circ}-55.3^{\circ} \mathrm{IL}$. The upper satellite traversed these same field lines about 22.5 minutes later at an altitude of 9840 $\mathrm{km}$. The Retarding Ion Mass Spectrometer (RIMS) on DE-1 recorded a region of significantly enhanced ion density at high altitude on SAR arc field lines (second panel) which appeared distinct from the plasmasphere as it is normally defined. This density enhancement of more than an order of magnitude was evident in both ions $\left(\mathrm{H}^{+}\right.$and $\left.\mathrm{He}^{+}\right)$being monitored during this particular pass. Enhancements in $\mathrm{O}^{+}, \mathrm{O}^{++}$, and $\mathrm{He}^{++}$have been observed colocated with SAR arc field lines o other occasions (not displayed). The correspondence between density enhancements in the plasmapause region and ionospheric electron temperature enhancements was also noted in an independent study /9/ correlating high and low altitude thermal plasma signatures. An invariant latitude-limited band of intense ELF hiss was observed by the Plasma Wave Instrument (PWI) as DE-1 flew across these field lines. Power spectral density at 2 $\mathrm{kHz}$ is plotted across the SAR arc region for the wave electric field (panel 3) and the wave magnetic field (panel 4). The magnitude of the enhancement in the hiss amplitude across the SAR arc region depends on the frequency considered.

An important signature in the energetic ions ( $\leq 17 \mathrm{kev}$ ) is illustrated in Figure 2. DE-2 traversed the 22 October 1981 SAR arc at approximately $900 \mathrm{~km}$ altitude between 7:00:00 and 7:00:44 UT. As in the previous example, the ionospheric electron temperature enhancement (upper panel) was taken to define the SAR arc field lines. Approximately 23 minutes later, DE-1 crossed these same field lines at an altitude of $6780 \mathrm{~km}$. During this particular pass observations of the low energy portion of the ring current $(\leq 17 \mathrm{kev})$ were available from the Energetic Ion Composition Spectrometer (EICS) on DE-1. $\mathrm{O}^{+}$(middle panel) and $\mathrm{H}^{+}$(lower panel) fluxes averaged over pitch angle are plotted as a function of ion energy in Figure 2 at the SAR arc peak and at the equatorward boundary. It is apparent that the low energy portion of the ring current is dominated by $\mathrm{O}^{+}$on SAR arc field lines. In fact the $\mathrm{O}^{+}$ flux is an order of magnitude greater than the $\mathrm{H}^{+}$flux in this region. Equally striking is the decrease in the $\mathrm{O}^{+}$flux equatorward of the SAR arc. There is a larger population of energetic $\mathrm{O}^{+}$ions over the peak than over the equatorward edge of the SAR arc. In contrast, the ring current $\mathrm{H}^{+}$distribution shows much less change when comparing observations taken over the peak and equatorward edge of the SAR arc.

\section{SUMMARY AND DISCUSSION}

When the position of the SAR arc, as defined by the ionospheric electron temperature enhancement, is mapped into the high altitude plasmapause region the following signatures are found to be coincident: (1) single or multi-peaked enhancements in the thermal plasma density which, at times, appear to be distinct from the plasmasphere, as it is normally defined, and which reflect the latitudinal structure of the SAR arc electron temperature enhancement, (2) invariant latitude-limited bands of ELF hiss intensified over values outside of the SAR arc region and (3) energetic (< $17 \mathrm{kev}$ ) ions dominated by $\mathrm{O}^{+}$with a significantly greater flux of $\mathrm{O}^{+}$on field lines that thread the SAR arc peak than equatorward of the SAR arc. These signatures are by no means unique, but are characteristic of the other data sets compiled for this study.

A ring current dominated by oxygen ions at energies below $17 \mathrm{kev}$ is a dramatically different environment than was envisioned in the early theories of SAR arc formation. Cornwall et al. $/ 2 /$ suggested Landau damping of proton cyclotron waves by thermal electrons as the source of energy for the SAR arc. This presupposes that protons are the major component of the ring current at resonant energies. If the ring current is dominated by oxygen at resonant energies $(20-30 \mathrm{kev})$, ion cyclotron wave growth rates would be significantly reduced and shifted to energies below the oxygen cyclotron frequency $/ 10 \%$. The possible predominance of oxygen ions at resonant energies may explain the puzzling lack of ion cyclotron wave observations on SAR arc field lines either because the waves do not grow to sufficient amplitudes or because they are shifted to frequencies below the measurement capabilities of DE- 1 and other satellites that have traversed this region.

Cole $/ 1 /$ proposed Coulomb collisions between ring current protons and thermal electrons as the energy source for SAR arc formation. Early attempts to evaluate the amount of energy available via this process were hampered by an inadequate knowledge of the ring current characteristics. Column electron heating rates were recently calculated $/ 7 /$ using measured energetic ion and thermal ion characteristics for several of the data sets compiled in the present study. Not only was sufficient energy transferred to the thermal electrons at high altitudes to support simultaneously observed SAR arc electron temperature enhancements in the ionosphere but the correct latitudinal variation in the heating rate was also reproduced, within the uncertainties of the calculation. Interestingly enough, in each case, the major portion of this energy was supplied by ring current oxygen ions. Thus, Coulomb collisions are capable of supplying significant energy to thermal electrons as suggested by Cole $/ 1 /$ but the source of this energy is ring current $\mathrm{O}^{+}$rather than protons as originally proposed.

The thermal ion density enhancements in the outer plasmasphere, which are colocated with the ionospheric SAR arc electron temperature enhancement, apparently contribute to the SAR arc process in two ways. Since the Coulomb heating rate of the thermal electrons is proportional to the product of the ring current and thermal electron density (sum of the thermal ion densities), these thermal ion density enhancements, in the presence of a smoothly varying ring current, serve to "select" the L shells where significant electron heating occurs and, in addition, are responsible for the production of multiple peaks in the electron temperature enhancement (multiple SAR arcs) at ionospheric heights. Also, these thermal ion density enhancements are probably responsible for the invariant latitude-limited bands of 
intense ELF hiss observed on these $\mathrm{L}$ shells. ELF hiss can be ducted by thermal density gradients. Ducting of plasmaspheric hiss in the growth region results in significantly enhanced wave amplitudes (e.g. /11/). These higher wave amplitudes may result in increased precipitation of electrons in cyclotron and Landau resonance with the waves. Observations by the DE-1 and -2 satellites of electron precipitation over SAR arcs are currently being examined to determine if this effect is significant. Such precipitation may be responsible for the trace emissions sometimes observed in connection with SAR arcs.

The observed high altitude particle and field signatures contribute to our understanding of the physical processes operating on SAR arc field lines and thus may ultimately serve to identify the mechanism or mechanisms responsible for supplying energy to the SAR arc.

\section{ACKNOWLEDGEMENTS}

We would like to acknowledge with gratitude the contributions of all of the instrument teams on DE-1 and -2 and Dr. D. W. Slater at Battelle Observatory in compiling the comprehensive data sets used in this SAR arc study. This work was supported by NASA under contract numbers NAS 5-28710, NAS 5-28712, NAS 5-28711, NAS 8-33982; grant numbers NAG 5-472, NAG 5-475, NAG 5-310, NGR-23-005-015; by AFGL under contract number FY 7121-84-N-306; and by NSF under grant number ATM 8508753.

\section{REFERENCES}

1. K. D. Cole, Stable auroral red arcs, sinks for energy of $D_{\mathrm{St}}$ main phase, J. Geophys. Res., 70, 1689 (1965)

2. J. M. Cornwall, F. V. Coroniti, and R. M. Thorne, Unified theory of SAR arc formation at the plasmapause, J. Geophys. Res., 76, 4428 (1971)

3. A. Hasegawa, and K. Mima, Anomalous transport produced by kinetic Alfven wave turbulence, J. Geophys. Res., 83, 1117 (1978)

4. M. H. Rees and R. G. Roble, Observations and theory of the formation of stable auroral red arcs, Rev. Geophys. Space Phys., 13, 201 (1975)

5. J. U. Kozyra, Observational and theoretical investigation of stable auroral red arcs and their magnetospheric energy source, Ph.D Thesis, Department of Atmospheric and Oceanic Sciences, University of Michigan, January 1986.

6. D. W. Slater, private communication (1982)

7. J. U. Kozyra, T. E. Cravens, A. F. Nagy, W. K. Peterson, and R. H. Comfort, The role of ring current $\mathrm{O}^{+}$in SAR arc formation, J. Geophys. Res., submitted (1986)

8. J. U. Kozyra, T. E. Cravens, A. F. Nagy, D. A. Gurnett, R. L. Huff, R. H. Comfort, J. H. Waite, Jr., W. K. Peterson, L. H. Brace, J. L. Burch, J. D. Winningham, Plasmaspheric signatures associated with stable auroral red arcs - new results from a coordinated DE-1 and -2 study, in preparation (1986)

9. J. L. Horwitz, L. H. Brace, R. H. Comfort, and C. R. Chappell, Dual spacecraft measurements of plasmasphere-ionosphere coupling, J. Geophys. Res., 91, 11203 (1986)

10. J. U. Kozyra, T. E. Cravens, A. F. Nagy, E. G. Fontheim and R. S. B. Ong, Effects of energetic heavy ions on electromagnetic ion cyclotron wave generation in the plasmapause region, J. Geophys. Res., $\underline{89}, 2217$ (1984)

11. C. Y. Huang, C. K. Goertz, and R. R. Anderson, A theoretical study of plasmaspheric hiss generation, $\underline{\text {. }}$ Geophys. Res., $\underline{88}, 7927$ (1983) 